\title{
Relação da produção de sementes de castanha-do-brasil com características morfométricas da copa e índices de competição
}

\author{
Helio Tonini(1), Paulo Emilio Kaminski(1) e Patricia da Costa(1) \\ (1)Embrapa Roraima, BR 174, Km 08, Distrito Industrial, CEP 69301-970 Boa Vista, RR. E-mail: helio@cpafrr.embapa.br, emilio@cpafrr.embrapa.br, \\ patrícia@cpafrr.embrapa.br
}

Resumo - O objetivo deste trabalho foi estudar a relação da produção de sementes de árvores nativas de castanha-dobrasil com características morfométricas da copa e índices de competição. Os dados foram coletados em florestas nativas no Sul do Estado de Roraima, com 88 árvores amostradas em diferentes classes de produtividade. Em cada árvore, foram medidas as variáveis dendrométricas e foram determinados os índices morfométricos da copa e os índices de competição, dependentes e independentes da distância. As relações entre a morfometria, os parâmetros da copa e os índices de competição com a produção de sementes foram obtidas com o coeficiente de correlação de Spearman e os testes de Kruskal-Wallis e Mann-Witney. Para inferir as relações entre as variáveis morfométricas da copa e o diâmetro do tronco, utilizou-se a análise de regressão linear pelo procedimento "stepwise". As árvores mais produtivas foram aquelas com posições superiores no dossel, que apresentam copas bem formadas, mais compridas e com menor relação altura/diâmetro. A competição teve pouco efeito sobre a produção de sementes, em árvores adultas que começam a produzir frutos somente após atingirem as posições superiores do dossel.

Termos para indexação: Bertholletia excelsa, Brasil, Amazônia, Roraima, produto florestal.

\section{Relationship of Brazil-nut seed yield to crown morphometric characteristics and competition indexes}

\begin{abstract}
The aim of this work was to evaluate the relationship of seed yield of Brazil-nut native trees to crown morphometric characteristics and competition indexes. The data were collected in 88 sample trees, in natural forests in the South of Roraima State, Brazil, regarding different productivity classes. In each tree, the dendrometric variables, the indexes of morphometry and competition (distance dependent and independent) were measured. The relationships between crown morphometric variables and competition indexes to seed yield were obtained through the Spearman correlation coefficient and Kruskal-Wallis and Mann-Whitney tests. A linear regression analysis, through the stepwise procedure, was used to infer crown relations among morphometric variables and the diameter at breast height. The most productive trees were those with upper canopy positions, wich showed well-formed and longer crowns, and smaller height/diameter relationships. Competition showed nonsignificant effect on seed yield, in adult trees that start to produce fruits only after reaching the upper canopy positions.
\end{abstract}

Index terms: Bertholletia excelsa, Brazil, Amazon, Roraima, forest product.

\section{Introdução}

As florestas com a presença de castanha-do-brasil (Bertholletia excelsa) cobrem uma superfície de aproximadamente 325 milhões de hectares naAmazônia (Stoian, 2004) e abrange a Venezuela, Colômbia, Peru, Bolívia e Guiana. As formações de florestas mais densas ocorrem no Brasil (Lorenzi, 2000).

A castanha-do-brasil pode ser considerada como espécie-chave para aliar a conservação ao desenvolvimento, por ser abundante na região Amazônica, colhida quase que exclusivamente em florestas naturais, ser explorada por diversas comunidades no curto prazo e a baixo custo, por apresentar sólida demanda de mercado, além de a coleta ser de baixo impacto ambiental, (Zuidema \& Boot, 2002).

Um dos grandes desafios para o extrativismo da castanha-do-brasil é o de aumentar a produção de frutos sem comprometer a sustentabilidade. Para frutos de alto valor econômico como os da castanha-dobrasil, um nível máximo de frutificação e uma mínima variação anual são fatores importantes para o sucesso comercial (Kainer et al., 2007). 
Para isso, é necessário desenvolver pesquisas que procurem quantificar a produção de frutos por árvore, e determinar quais são os fatores que influenciam a variabilidade da produção individual em diferentes regiões da Amazônia.

Estudos de Viana et al. (1998), Zuidema \& Boot (2002), Wadt et al. (2005) e Kainer et al. (2006, 2007) observaram que a produção de frutos de árvores de castanha-do-brasil é muito variável, e os principais fatores que determinam essa variabilidade são: tamanho da árvore (principalmente o diâmetro do tronco - DAP); atributos da copa, como posição sociológica e infestação por cipós; variações temporais inerentes a própria planta; fatores climáticos, como a precipitação; nutrição (níveis de fósforo), além de fatores genéticos e interações com polinizadores, predadores e dispersores. Outros fatores que podem ter alguma influência, e ainda não foram abordados, referem-se à morfometria da copa e à competição.

Segundo Hasenauer (1997), a morfometria de uma árvore e as variáveis derivadas são utilizadas para inferir sobre relações interdimensionais, reconstituir o espaço ocupado por cada árvore, a sua estabilidade, vitalidade e produtividade, bem como julgar o grau de concorrência a que cada indivíduo foi ou está sujeito.

Naciência florestal, as pesquisas e o desenvolvimento de índices de competição têm sido aplicados principalmente na prognose do crescimento em plantios florestais ou grupos de espécies em florestas naturais. O status competitivo de uma árvore é descrito por medidas de competição independentes ou dependentes da distância. Índices independentes da distância distinguem-se dos dependentes por utilizarem medidas não espaciais, baseadas na distribuição dos tamanhos das árvores em uma determinada área. Nos índices dependentes da distância (espaciais), os competidores são identificados baseados em seu tamanho e distância em relação à árvore-objetivo (Wimberly \& Bare, 1996).

O objetivo deste trabalho foi estudar a relação da produção de sementes de árvores nativas de castanhado-brasil com características morfométricas da copa e índices de competição.

\section{Material e Métodos}

Os dados foram coletados em florestas com ocorrência natural de castanha-do-brasil no sul do Estado de Roraima, no Município de Caracarai, nas coordenadas $1^{\circ} 48^{\prime} 58^{\prime \prime} \mathrm{N}$ e $61^{\circ} 7^{\prime} 41^{\prime \prime} \mathrm{W}$, distante $135 \mathrm{~km}$ de Boa Vista.

Aárea em estudo apresenta relevo plano a ondulado, com vegetação predominante considerada floresta tropical úmida (Brasil, 1975). O clima na região é classificado como Ami (tropical chuvoso, com pequeno período de seca), com precipitação média anual entre 1.700-2.000 mm. O período chuvoso ocorre com maior freqüência de abril a agosto com totais mensais superiores a $100 \mathrm{~mm}$. A partir de setembro ocorre uma sensível redução, e o período característico ocorre de novembro a março. A temperatura média anual é de $27^{\circ} \mathrm{C}$ (Fundação..., 1993).

Para a realização deste estudo foi monitorada a produção de frutos e de sementes de 239 árvores, em duas ocasiões: no meio e no final do período de queda dos frutos (maio e julho), durante o ano de 2007. Em cada árvore, foi feita a contagem dos frutos e a pesagem das sementes, em balança de gancho digital, com precisão de $50 \mathrm{~g}$.

Após a obtenção dos dados de produção, as árvores foram agrupadas em quatro categorias de produtividade, definidas da seguinte forma: nenhuma, baixa (de 0,1 a $5 \mathrm{~kg}$ ), média (de 5 a $10 \mathrm{~kg}$ ) e alta (produção superior a $10 \mathrm{~kg}$ de sementes por árvore). A partir desta classificação, foram selecionadas, aleatoriamente, 88 árvores-amostras estratificadas em classes de produtividade.

\section{Variáveis morfométricas}

Em cada árvore foram medidos: o DAP, a altura total (h) e a altura de inserção da copa (hic), tomadas com o Vertex. Foram calculados: o comprimento da copa (L) - definido como a diferença entre $h$ e hic e o diâmetro da copa (Dc) - tomando-se quatro raios nas direções $\mathrm{N}-\mathrm{S}$ e $\mathrm{L}-\mathrm{O}$, usando uma bússola, um clinômetro e o Vertex, a fim de determinar as distâncias (raios).

As variáveis da copa estudadas foram: posição sociológica, forma, e presença de cipós. A posição sociológica e a forma da copa foram obtidas pelo índice de Dawkins, modificado conforme Wadt et al. (2005) e Kainer et al. (2006), em que as copas foram classificadas em relação à forma: completa ou circular, irregular, meia copa, menos do que metade da copa, um ou menos galhos. Em relação à posição sociológica, foram classificadas como: dominante, co-dominante, intermediária e suprimida. 
A presença de cipós na copa foi avaliada visualmente utilizando-se os critérios empregados por Wadt et al. (2005) e Kainer et al. (2006): copa sem cipó; copa com menos $25 \%$ de infestação por cipós; copas com 25 a $75 \%$ de infestação e copa acima de $75 \%$ de infestação por cipós.

Os índices morfométricos, baseados no tamanho e na morfologia da árvore, utilizados neste estudo foram: proporção de copa (PC); relação altura/diâmetro ou grau de Esbeltez (GE), índice de saliência (IS), índice de abrangência (IA) e formal da copa (FC), expressados por: $\mathrm{PC}=100 \mathrm{~L} / \mathrm{h}, \mathrm{GE}=\mathrm{h} / \mathrm{d}, \mathrm{IS}=\mathrm{Dc} / \mathrm{d}$, $\mathrm{IA}=\mathrm{Dc} / \mathrm{h}, \mathrm{FC}=\mathrm{Dc} / \mathrm{L}$, em que: $\mathrm{L}$ é o comprimento da copa (m); h, altura total (m); d, diâmetro do tronco $(\mathrm{cm})$; Dc, diâmetro da copa $(\mathrm{m})$.

\section{Índices de competição}

Para avaliar a influência do status competitivo sobre a produção de sementes de castanheira-dobrasil, foram testados 17 índices de competição espaciais e não espaciais. O número de competidores foi definido em função do raio da copa, e foram considerados competidores todos os indivíduos localizados à distância equivalente a uma ou duas vezes o raio médio da copa, com diâmetro igual ou superior a $10 \mathrm{~cm}$. Por este critério, cada índice foi calculado duas vezes, pelo teste de duas distâncias, para a definição do número de competidores.

Para cada competidor mediu-se o diâmetro do tronco, a distância em relação à árvore-objetivo, e determinou-se a posição sociológica. Os índices foram selecionados com base nos estudos de Hegyi (1974), Lorimer (1983), Tomé \& Burkhart (1989) e Moravie et al. (1999).

\section{Índices de competição não espaciais}

Soma das áreas basais dos competidores $(\mathrm{G})$; soma das áreas basais dos competidores mais grossos do que a árvore-objetivo $\left(\mathrm{G}_{\mathrm{g}}\right)$; soma das áreas basais dos competidores de mesma posição sociológica em relação à árvore-objetivo $\left(\mathrm{G}_{\mathrm{p}}\right)$; soma dos diâmetros dos competidores relativos (Sdr), expressados por: $\Sigma \mathrm{d}_{\mathrm{c}} / \mathrm{d}_{\mathrm{o}}$, em que: $\mathrm{d}_{\mathrm{c}}$ é o diâmetro dos competidores; $\mathrm{d}_{\mathrm{o}}$, o diâmetro da árvore-objetivo; $\mathrm{Sdr}_{\mathrm{g}}$, a soma dos diâmetros dos competidores relativos mais grossos do que a árvore-objetivo; $\operatorname{Sdr}_{\mathrm{p}}$, a soma dos diâmetros dos competidores relativos de mesma posição sociológica que a árvore-objetivo.
IC: índices propostos por Tomé \& Burkhart (1989), expressados por: $\mathrm{IC}=\mathrm{d}_{\mathrm{o}} / \mathrm{d}_{\mathrm{m}}, \mathrm{IC}_{2}=\mathrm{d}_{\mathrm{o}} / \mathrm{d}_{\max }, \mathrm{IC}_{3}=\mathrm{d}_{\mathrm{o}} / \mathrm{d}_{\mathrm{dom}}$, em que: $d_{o}$ é o diâmetro da árvore-objetivo; $d_{m}, o$ diâmetro médio dos competidores; $\mathrm{d}_{\max }$, o diâmetro máximo dos competidores; $\mathrm{d}_{\mathrm{dom}}$, o diâmetro médio das árvores dominantes e co-dominantes.

\section{Índices espaciais}

Gdist: área basal ponderada pela distância dos competidores, que é expressada por: $\Sigma \mathrm{g}_{\mathrm{c}} /$ Dist $_{\mathrm{c}}$, em que: $\mathrm{g}_{\mathrm{c}}$ é a área basal dos competidores; $\mathrm{d}_{\mathrm{c}}$, a distância

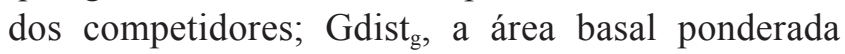
pela distância dos competidores mais grossos do que a árvore-objetivo; Gdist ${ }_{\mathrm{p}}$, a área basal ponderada pela distância dos competidores de mesma posição sociológica que a árvore-objetivo.

Sddist: soma dos diâmetros ponderada pela distância dos competidores, que é expressada por: $\Sigma \mathrm{d}_{\mathrm{c}} /$ Dist $_{\mathrm{c}}$, em que: $d_{c}$ é o diâmetro dos competidores; Dist $t_{k}$, a distância dos competidores; Sddist $\mathrm{g}_{\mathrm{g}}$ a soma dos diâmetros, ponderada pela distância dos competidores mais grossos do que a árvore-objetivo; Sddist $t_{p}$, a soma dos diâmetros, ponderada pela distância dos competidores de mesma posição sociológica, em relação à árvore-objetivo.

$\mathrm{IH}$ : índice de Hegyi, expressado por: $\mathrm{IH}=\left(\mathrm{d}_{\mathrm{d}} / \mathrm{d}_{\mathrm{o}}\right)(1 /$ dist $)$, em que: $\mathrm{d}_{\mathrm{o}}$ é o diâmetro da árvore-objetivo; $\mathrm{d}_{\mathrm{c}}$, o diâmetro do competidor; dist, a distância do competidor em relação à árvore-objetivo.

IHm: índice de Hegyi modificado, expressado por: $\mathrm{IHm}=\left(\mathrm{d}_{\mathrm{c}} / \mathrm{d}_{\mathrm{o}}\right)(\mathrm{dist} / \mathrm{R})$, em que: $\mathrm{d}_{\mathrm{o}}$ é o diâmetro da árvore-objetivo; $\mathrm{d}_{\mathrm{m}}$, o diâmetro do competidor; dist, a distância do competidor em relação à árvore-objetivo; $\mathrm{R}$, o raio de amostragem.

Para avaliar a normalidade dos dados de produção de sementes utilizou-se o teste de Kolmogorov-Smirnov. A partir dos resultados obtidos foi necessário utilizar testes não paramétricos para inferir sobre as relações entre as variáveis estudadas.

Empregou-se o coeficiente de correlação de Spearman, para verificar a magnitude dos efeitos entre as variáveis morfométricas e a produção de sementes. Com o estudo da correlação, foram selecionadas as variáveis que apresentaram correlações significativas, a 5\% de probabilidade.

O coeficiente de determinação, ou seja, o quadrado do coeficiente de correlação foi utilizado para medir o somatório da variabilidade na produção de sementes, explicada pelas variáveis morfométricas.

Pesq. agropec. bras., Brasília, v.43, n.11, p.1509-1516, nov. 2008 
Para verificar se existiam diferenças morfométricas entre árvores, nas diferentes classes de produtividade, utilizou-se o teste de Kruskal-Wallis. Para identificar contrastes entre grupos, utilizou-se o teste de MannWitney, com a aplicação da correção de Bonferroni para comparações múltiplas.

Uma vez que o diâmetro do tronco é a variável mais freqüentemente medida em inventários florestais (Machado \& Figueiredo Filho, 2003), apresenta relação direta com variáveis da copa, como o diâmetro e a superfície (Nutto, 2001), e se correlaciona positivamente com a produção de frutos e de sementes de castanha-do-brasil (Zuidema, 2003; Wadt et al., 2005; Kainer et al., 2007), foi necessário inferir sobre as relações entre as variáveis morfométricas e o diâmetro do tronco (DAP). Para isso, utilizou-se a análise de regressão linear pelo procedimento estatístico "stepwise", no qual a variável potencial independente utilizada foi $o$ diâmetro do tronco na sua forma simples, quadrática, cúbica, inversa e logarítmica $\left(\mathrm{d}, \mathrm{d}^{2}, \mathrm{~d}^{3}, 1 / \mathrm{d}, 1 / \mathrm{d}^{2}, 1 / \mathrm{d}^{3}\right.$, $\ln \mathrm{d}, \ln ^{2} \mathrm{~d}$ ), selecionadas com significância a $5 \%$ de probabilidade.

A eficiência dos modelos foi verificada pelo coeficiente de determinação ajustado $\left(\mathrm{R}^{2}{ }_{\mathrm{aj}}\right)$, erro-padrão de estimativa em percentagem (Syx\%), recalculado para unidades aritméticas, no caso de equações logarítmicas, valor e significância de $\mathrm{F}$ para o modelo, valor e significância de t para os coeficientes e a análise da distribuição dos resíduos.

\section{Resultados e Discussão}

Em média, uma árvore de castanha-do-brasil produziu $25 \pm 42,6$ frutos, com produção de $3,43 \pm 6,06 \mathrm{~kg}$ de sementes por árvore. Esses valores são inferiores aos observados por diferentes pesquisadores em reservas extrativistas no Estado do Acre. Viana et al. (1998) observaram produção média por árvore de $24 \mathrm{~kg}$ com um mínimo de $1,5 \mathrm{~kg}$ e um máximo de $105 \mathrm{~kg}$. Wadt et al. (2005) observaram no ano de 2002 produção média de 10,28 $\mathrm{kg}$ por árvore, em 140 árvores amostradas com DAP mínimo $\geq 50 \mathrm{~cm}$, e Kainer et al. (2007) monitoraram 140 árvores durante cinco anos consecutivos e obtiveram número médio de frutos, coletados por ano, de 66,2 \pm 98 e 9,3 kg por árvore.

Em dois locais na Bolívia, Zuidema \& Boot (2002) obtiveram produção média, por árvore e por ano, de $184 \pm 129$ e $139 \pm 104$ frutos, ao monitorar 93 indivíduos durante cinco e três anos consecutivos, respectivamente.

As diferenças de produção podem ser explicadas, pelo menos em parte, por diferenças na metodologia empregada. Os trabalhos de Viana et al. (1998) e Zuidema \& Boot (2002) foram realizados com o auxílio dos castanheiros que, normalmente, evitam as árvores menos produtivas. Neste trabalho e no de Kainer et al. (2007), as árvores foram selecionadas aleatoriamente sem a interferência do castanheiro.

A aplicação do teste de Kolmogorov-Smirnov indicou que os dados de produção de sementes e número de frutos diferiram significativamente da distribuição normal $(Z=1,981 ; p=0,001$ e $Z=1,84$; $p=0,002)$. Para avaliar a correlação entre a produção de sementes, a morfometria e copa das árvores, aplicouse o coeficiente de correlação de Spearman (Tabela 1).

Observou-se relação significativa entre a produção de sementes com comprimento, diâmetro, forma e exposição da copa, ou posição sociológica, bem como, com os índices morfométricos: IA e GE.

Entre as variáveis da copa: comprimento, diâmetro e posição explicaram a maior variabilidade na produção de sementes com $12,9,15,1$ e $14,6 \%$, respectivamente. No entanto, o DAP foi a variável que melhor explicou essa variação com $18,5 \%$.

O tamanho dos efeitos, medido pelo coeficiente de correlação, pode ser considerado médio, segundo Cohen (1992), com valores em torno de 0,3.

Wadt et al. (2005) observaram que quanto maior o diâmetro do tronco, melhor a posição sociológica e a forma da copa, e maior a produção de sementes de castanha-do-brasil. As correlações apresentadas na

Tabela 1. Correlação e coeficiente de determinação entre a produção de sementes (PS) de árvores de castanha-do-brasil e a sua morfometria ${ }^{(1)}$.

\begin{tabular}{|c|c|c|c|c|c|c|c|c|}
\hline Variável & $\mathrm{L}$ & $\mathrm{D}_{\mathrm{c}}$ & $\mathrm{FC}$ & PS & $\mathrm{PC}$ & IA & GE & DAP \\
\hline$\overline{\mathrm{PS}}$ & $0,36^{* *}$ & $0,39 * *$ & $-0,22 *$ & $-0,24^{*}$ & $0,38^{*}$ & $0,30^{*}$ & $-0,31^{*}$ & 0,43 \\
\hline $\mathrm{R}^{2}$ & 12,90 & 15,10 & 4,80 & 5,30 & 14,60 & 9,10 & 9,60 & 18,50 \\
\hline DAP & $0,61 * *$ & $0,77 * *$ & $-0,43 * *$ & $-0,43 * *$ & $0,49 * *$ & $0,58 * *$ & $-0,85^{* *}$ & 1,00 \\
\hline $\mathrm{R}^{2}$ & 37,10 & 59,10 & 18,50 & 17,60 & 24,40 & 33,70 & 73,10 & 100,00 \\
\hline
\end{tabular}


Tabela 1 corroboram essas observações, no entanto, em Wadt et al. (2005), a proporção da variação na produção de sementes explicada pelo DAP foi bastante superior $\left(\mathrm{R}^{2}=37,2 \%\right)$.

Foram observadas diferenças significativas $(\mathrm{H}=9,91$; $\mathrm{p}=0,04 ; \mathrm{H}=14,21 ; \mathrm{p}=0,03$, respectivamente) entre a produção de sementes nas diferentes categorias de forma da copa e exposição da copa. Para identificar essas diferenças, aplicou-se o teste de Mann-Witney.

Somente as árvores dominantes e co-dominantes produziram frutos, e a produção foi superior para as árvores dominantes $(\mathrm{U}=61,0 ; \mathrm{p}=0,003)$. Pode-se constatar que o status reprodutivo da espécie é fortemente correlacionado com a posição no dossel e a grande maioria das árvores reprodutivas são emergentes, o que concorda com Zuidema (2003), ao afirmar que o pleno desenvolvimento da copa, em árvores de castanha-dobrasil, só é observado em árvores presentes nas camadas emergentes do dossel, onde não existem limitações para o pleno desenvolvimento lateral da copa.

A forma da copa teve influência significativa na produção de sementes $(H=9,98 ; p=0,042)$, tendo sido aplicado o teste de Mann-Witney para detectar diferenças entre grupos. Com a aplicação da correção de Bonferroni, para duas comparações, foram consideradas significativas as diferenças a $2,5 \%$ de probabilidade.

A produção de sementes não diferiu para árvores com copas circulares completas ou irregulares $(U=394,5$; $\mathrm{p}=0,17)$, o que concorda com Kainer et al. (2007). Árvores com copa completa, de forma circular ou irregular, produziram, em média, 7,4 e $10,1 \mathrm{~kg}$ de sementes por árvore. Árvores com copas pobres (meia copa ou menos) produziram, em média, 3,5 e $1,8 \mathrm{~kg}$ por árvore, o que difere das árvores com copas bem formadas $(U=456 ; p=0,02)$.

A comparação entre as variáveis morfométricas, nas diferentes classes de produção, permitiu observar diferenças significativas entre as árvores localizadas na classe de maior produção e todas as demais para as variáveis: proporção da copa e grau de Esbeltez. Com a aplicação da correção de Bonferroni, para três comparações, adotou-se nível de $0,017 \%$ de significância.

As árvores mais produtivas apresentaram uma proporção de copa média de $47,3 \%$, o que difere das árvores com produção média $(40,3 \% ; \mathrm{U}=59 ; \mathrm{p}=0.005)$, baixa $(40,3 \%, U=226 ; p=0,015)$ e nula $(32,4 \%, U=74$; $\mathrm{p}=0,001)$. Hasenauer \& Monserud (1996) consideram a proporção de copa uma medida dimensional muito útil, uma vez que o crescimento apical, cambial e radicular são controlados por fotossintatos e hormônios produzidos na copa.

Em relação ao grau de Esbeltez, as árvores mais produtivas apresentaram os menores valores com média de 0,42 , tendo diferido das árvores com produção média $(0,52 ; \mathrm{U}=52 ; \mathrm{p}=0,007 ; \mathrm{r}=-0,45)$, baixa $(0,51 ; \mathrm{U}=2,14$; $\mathrm{p}=0,009)$ e nula $(0,73 ; \mathrm{U}=112 ; \mathrm{p}=0,002)$.

Pode-se concluir que as árvores mais produtivas possuem copas mais compridas e são mais robustas (menos delgadas). A diminuição do grau de Esbeltez, com o aumento da classe de produtividade, indica que nas árvores mais produtivas, as taxas de crescimento em diâmetro são maiores do que em altura.

Segundo Zuidema (2003), na estratégia de buscar atingir o dossel o mais rápido possível, o crescimento das árvores jovens de castanha-do-brasil é direcionado mais para a altura do que para o diâmetro, o que resulta em árvores com maior relação altura-diâmetro. Após atingir a maturidade e as posições superiores do dossel, ocorre sensível redução no incremento em altura e um maior incremento diamétrico, o que faz com que a relação altura/diâmetro decresça com o aumento do tamanho da árvore, que está diretamente relacionado ao status reprodutivo da espécie.

Kainer et al. (2007), ao estudar os fatores que afetam a produção de frutos e sementes de árvores de castanheirado-brasil, observaram que o diâmetro do tronco foi a variável de maior importância e explicou $56,5 \%$ da variação da produção de frutos.

Neste trabalho, foi observada correlação significativa entre o DAP e a produção de sementes $(r=0,349$; $\mathrm{p}=0,001)$, e com todas as variáveis morfométricas (Tabela 1). Foi possível estudar a relação dessas variáveis com a modificação no tamanho das árvores por análise de regressão.

Os modelos obtidos com o procedimento "stepwise", para estimar a proporção da copa (PC) e o grau de Esbeltez (GE), em função do DAP, foram: $\ln \mathrm{PC}=2,838$ $+0,042 \ln ^{2} \operatorname{DAP}\left(\mathrm{R}_{\text {aj }}^{2}=0,28 ; \operatorname{Syx} \%=25,2 ; \mathrm{p}=0,001\right)$; $\mathrm{GE}=2,335-0,407 \ln \mathrm{DAP}\left(\mathrm{R}^{2}{ }_{\text {aj }}=0,88 ; \mathrm{Syx} \%=15,8\right.$; $\mathrm{p}=0,001)$.

Na Figura $1 \mathrm{~A}$, observa-se que as árvores de tamanho intermediário - com DAP entre 80 e $150 \mathrm{~cm}$ - foram as mais produtivas, resultado também observado por Kainer etal.(2007). Para o grau de Esbeltez (Figura 1 B), o modelo obtido pode ser considerado satisfatório com aproximadamente $90 \%$ da variação explicada apenas pelo diâmetro do tronco. Já para a proporção de copa, 
não foi possível obter boas estimativas apenas com o DAP como variável independente. No entanto, por ser significativo, o modelo representa uma tendência.

A proporção de copa tende a aumentar com o diâmetro do tronco (Figura $1 \mathrm{C}$ ), ou seja, quanto maior o diâmetro, menor a altura de inserção dos galhos, e maior o comprimento da copa. Esse resultado concorda com Durlo \& Denardi (1998), que consideraram a proporção de copa como um bom indicador da capacidade da árvore em utilizar os recursos disponíveis para o crescimento. O grau de Esbeltez apresentou tendência inversa.

Para avaliar o efeito da competição sobre a produção de sementes, utilizou-se o procedimento "stepwise", em que as variáveis independentes utilizadas foram os diferentes índices de competição em sua forma simples e logarítmica. Para cumprir os pré-requisitos da análise de regressão linear, as árvores sem nenhuma produção foram retiradas e a variável dependente (produção de sementes) foi convertida para a escala logarítmica, o que levou os dados a distribuição normal $(\mathrm{Z}=0,84$; $\mathrm{p}=0,47)$ e a homogeneidade de variância $\mathrm{F}_{(2,65)}=16,05$, não significativo (teste de Levene).

O índice de competição selecionado foi o de Hegyi, com os competidores definidos a uma distância igual a duas vezes o raio médio da copa. Esse índice é um dos mais utilizados na modelagem do crescimento de árvores individuais, e é calculado pela soma das razões entre o diâmetro do competidor e da árvore-objetivo, ponderada pela distância eles. Dessa forma, atribuise um peso maior a competidores mais próximos, e grandes valores indicam maior competição.

Moravie et al. (1999), ao rever vários estudos sobre aumentos no poder de predição pela adoção de índices dependentes da distância, concluíram que nenhum índice, dependente ou independente da distância, tem-semostrado superior aos demais. Ou seja, a habilidade preditiva de determinado índice de competição, normalmente, depende da espécie e da situação estudada.

O modelo obtido para expressar a relação entre a produção de sementes e a competição, apesar de significativo, explicou menos do que $10 \%$ da variação na produção de sementes (Figura 2).

A competição apresentou pouco efeito sobre a produção de sementes para árvores adultas e dominantes, que começam a produzir frutos somente após atingir as posições superiores do dossel. No entanto, observou-se uma tendência de diminuição da produção de sementes com o aumento da competição.
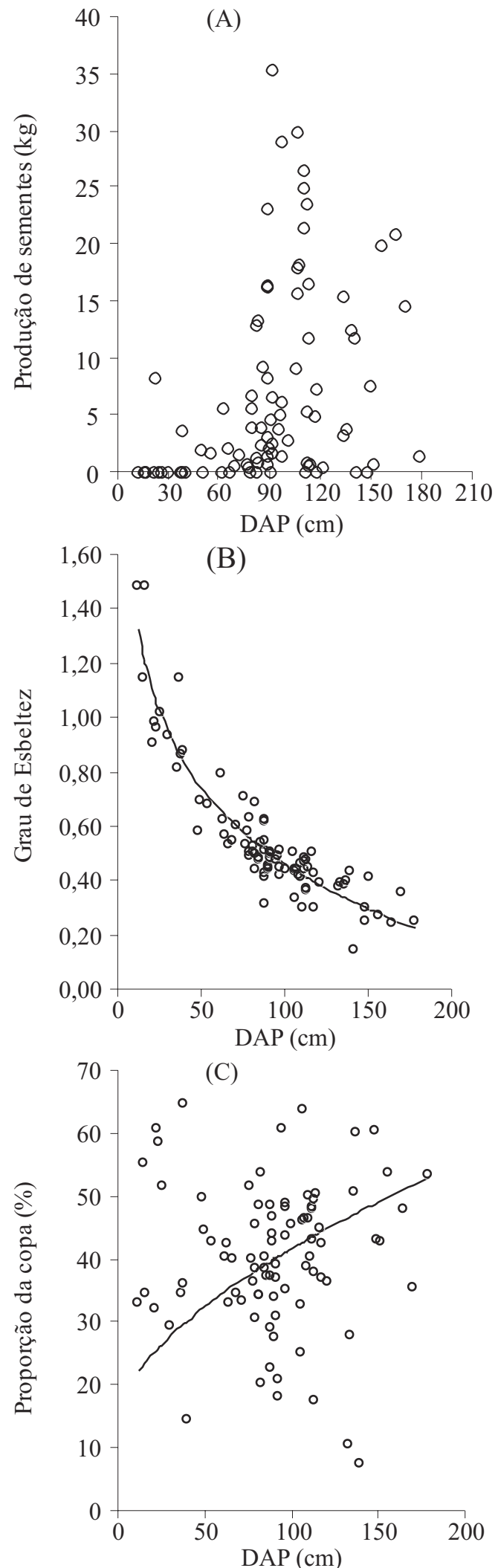

Figura 1. Relação entre diâmetro do tronco (DAP) e: produção de sementes (A); grau de Esbeltez (B); proporção da copa (C). 


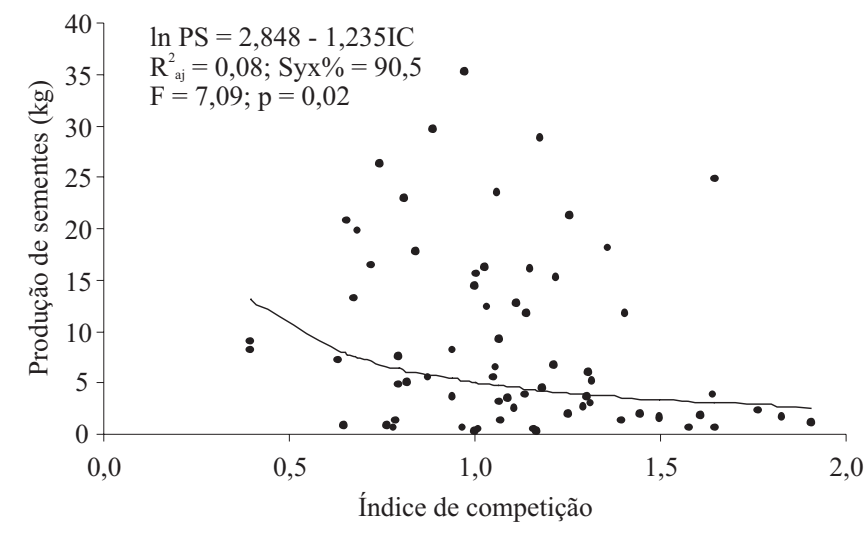

Figura 2. Relação entre produção de sementes e competição em árvores de castanha-do-brasil.

\section{Conclusões}

1. A posição sociológica e a forma da copa têm influência sobre a produção de sementes; arvores nas posições superiores do dossel e com copas bem formadas, de forma circular ou irregulares, são mais produtivas.

2. As árvores mais produtivas apresentam copas mais compridas e menor relação altura/diâmetro.

3. O grau de Esbeltez pode ser estimado com boa precisão a partir do diâmetro do tronco.

4. A competição apresenta pouco efeito sobre a produção de sementes em árvores adultas; no entanto, há tendência de redução da produção de sementes com o aumento da competição.

\section{Agradecimentos}

Ao Conselho Nacional de Desenvolvimento Científico e Tecnológico, pelo auxílio financeiro; a José Adebaldo Telles e Anchieta Moreira da Costa, pelo auxílio e dedicação na coleta dos dados.

\section{Referências}

BRASIL. Departamento Nacional da Produção Mineral. Projeto Radambrasil: Folha NA 20, Boa Vista e parte das folhas NA 21, Tumucumaque, NB 20, Roraima e NB 21; geologia, geomorfologia, pedologia, vegetação e uso potencial da terra. Rio de Janeiro: DNPM, 1975. 428p. (Levantamento de Recursos Naturais, 8).

COHEN, J. A power primer. Psychological Bulletin, v.112, p.155-159, 1992.
DURLO, M.A.; DENARDI, L. Morfometria de Cabralea canjerana, em mata secundária nativa do Rio Grande do Sul. Ciência Florestal, v.8, p.55-56, 1998.

FUNDAÇÃODOMEIOAMBIENTECIÊNCIAETECNOLOGIA DO ESTADO DE RORAIMA. O Brasil do hemisfério norte: diagnóstico científico e tecnológico para o desenvolvimento. Boa Vista: Ambtec, 1993. 512p.

HASENAUER, H. Dimensional relationships of open-grown trees in Austria. Forest Ecology and Management, v.96, p.197-206, 1997.

HASENAUER, H.; MONSERUD, R.A. A crown ratio model for Austrian forests. Forest Ecology and Management, v.84, p.49-60, 1996.

HEGYI, F. A simulation model for managing jack-pine stands. In: FRIES, G. Growth models for tree and stand population. Stockolm: Royal College of Forestry, 1974. p.74-90.

KAINER, K.A.; WADT, L.H.O.; GOMES-SILVA, D.A.P.; CAPANU, M. Liana loads and their association with Bertholletia excelsa fruit and nut production, diameter growth and crown atributes. Journal of Tropical Ecology, v.22, p.147-154, 2006.

KAINER, K.A.; WADT, L.H.O.; STAUDHAMMER, C.L. Explaining variation in Brazil nut fruit production. Forest Ecology and Management, v.250, p.244-255, 2007.

LORENZI, H. Árvores brasileiras: manual de identificação e cultivo de plantas arbóreas nativas do Brasil. 4.ed. São Paulo: Instituto Plantarum, 2000. v.1. 384p.

LORIMER, C.G. Test of age-independent competition index for individual trees in natural hardwood stands. Forest Ecology and Management, v.6, p.343-360, 1983.

MACHADO, S.A.; FIQUEIREDO FILHO, A. Dendrometria. Curitiba: UFPR, 2003. 309p.

MORAVIE, M.A.; DURAND, M.; HOULLIER, F. Ecological meaning and predictive ability of social status vigour and competition indices in a tropical rainforest (India). Forest Ecology and Management, v.117, p.221-240, 1999.

NUTTO, L. Manejo do crescimento diamétrico de Araucaria angustifolia (Bert.) O. Ktze. baseado na árvore individual. Ciência Florestal, v.11, p.9-25, 2001.

STOIAN, D. Cosechando lo que cae: la economía de la castaña Bertholletia excelsa H.B.K) em la amazônia boliviana. In: ALEXIADES, M.N.; SHANLEY, P. (Ed.). Productos forestales, medios de subsistencia y conservación de productos forestales no maderables. Borgor: Cifor, 2004. v.3. p.89-116.

TOMÉ, M.; BURKHART, H.E. Distance-dependent competition measures for predicting growth of individual trees. Forest Science, v.35, p.816-831, 1989.

VIANA, V.M.; MELLO, R.A.; MORAES, L.M.; MENDES, N.T. Ecologia e manejo de populações de castanha-do-pará em reservas extrativistas, Xapuri, Estado do Acre. In: GASCON, 
C.; MOUTINHO, P. (Ed.). Floresta Amazônica: dinâmica, regeneração e manejo. Manaus: Inpa, 1998. p.277-292.

WADT, L.H.O.; KAINER, K.A.; GOMES-SILVA, D.A.P. Population structure and nut yield of a Bertholletia excelsa stand in Southwestern Amazonia. Forest Ecology and Management, v.211, p.371-384, 2005.

WIMBERLY, M.C.; BARE, B.B. Distance-dependent and distanceindependent models of Douglas-fir and western hemlock basal area growth following silvicultural treatment. Forest Ecology and Management, v.89, p.1-11, 1996.

ZUIDEMA, P.A. Ecology and management of the Brazil nut tree (Bertholletia excelsa). Riberalta: Promab, 2003. 111p. (Promab Scientific Series, 6).

ZUIDEMA, P.A.; BOOT, R.G.A. Demography of the Brazil nut tree (Bertholletia excelsa) in the Bolivian Amazon: impact of seed extraction on recruitment and population dynamics. Journal of Tropical Ecology, v.18, p.1-31, 2002.

Recebido em 10 de julho de 2008 e aprovado em 27 de outubro de 2008 